\title{
Lung function after exposure to barley dust
}

\author{
P E MCCARTHY, A E COCKCROFT, AND M MCDERMOTT \\ From the MRC Pneumoconiosis Unit, Llandough Hospital, Penarth, S Glamorgan, UK
}

ABSTRACT Six men handling barley were followed up over two working days and their flow volume curves were measured every two hours. All experienced falls in ventilatory capacity of up to $800 \mathrm{ml}$ with changes in the shape of the flow volume curve in five men. Five volunteers not previously exposed to barley dust sat in a silo for two hours. Decreases in ventilatory capacity ranging from $200 \mathrm{ml}$ to $800 \mathrm{ml}$ were found, with recovery taking up to 72 hours. All subjects had decreases in flow at $50 \%$ vital capacity but little or no change in flow at $75 \%$ vital capacity. In three subjects there was a drop in specific conductance that lasted for less than 24 hours. Two subjects were similarly exposed on a second occasion when transfer factor for carbon monoxide was measured in addition to flow volume curves. There was a decrease in transfer factor but no change in transfer factor per unit volume (Kco).

In recent years several studies have reported respiratory symptoms and reduced lung function in grain workers compared with control groups. ${ }^{1-3}$

The present study was carried out at a small dock in Gloucestershire. We have previously reported the prevalence of grain related symptoms and their association with smoking, atopic status, and ventilatory capacity, together with the changes in ventilatory capacity that occurred in a group of dockworkers during a shift handling barley and in six normal unexposed subjects after exposure to barley dust. ${ }^{4}$ In this paper we present data on forced expiratory flow rates in the same group of people together with other indices of lung function in the previously unexposed subjects.

\section{Survey of dockworkers}

Various cargoes are handled at the dock, including grains such as barley, wheat, and maize which are stored in two large silos and loaded and unloaded into ships using both machinery and manual labour. The men are exposed to grain dust only intermittently and mainly in the open air. The workforce is stable, since dock work tends to be a family occupation.

Eighty men of a possible 85 took part in the study. They all completed the MRC respiratory symptom questionnaire with additional questions about work related symptoms and performed forced expiratory

Received 23 January 1984

Accepted 2 April 1984 flow manoeuvres into a McDermott digital incremental spirometer. ${ }^{5}$ The indices obtained from the curves were forced expiratory volume in one second $\left(\mathrm{FEV}_{1}\right)$, forced vital capacity (FVC), peak flow (PF), and flows at $50 \%$ and $75 \%$ of the expired vital capacity $\left(\dot{\mathrm{V}}_{50}\right.$ and $\left.\dot{\mathrm{V}}_{75}\right)$.

\section{RESULTS}

Sixty one of the 80 men complained of respiratory symptoms after handling certain cargoes including barley, with barley being the only cargo mentioned by 14 . The FEV, and FVC were greater than the $80 \%$ predicted $^{6}$ in all but seven men, who were all smokers or ex-smokers and had given questionnaire answers indicative of chronic bronchitis or asthma.

Since prediction equations for flow rates are less well established, a lower limit of $70 \%$ predicted $^{6}$ was used as the criterion for "normality." The table shows the numbers above and below $70 \%$ predicted for smokers, ex-smokers, and non-smokers. The peak flows were close to predicted but the $\dot{V}_{50}$ and $\dot{\mathrm{V}}_{75}$ showed a different picture. Thirty three men had a low $\dot{V}_{50}$ and 59 a low $\dot{V}_{75}$. For both indices the "subnormal" values occurred more often in the smokers and ex-smokers $\left(\mathrm{X}_{\mathrm{df2}}^{2}=7.62, \mathrm{p}<0.01\right.$ for $\dot{\mathrm{V}}_{\text {so }}, \mathrm{X}_{\mathrm{df2}}=13.64, \mathrm{p}<0.005$ for $\left.\dot{\mathrm{V}}_{75}\right)$. The low flows did not occur more often in men who complained of respiratory symptoms associated with their work.

\section{Across shift measurements}

Flow volume curves were obtained over two consecutive days on six dockworkers loading barley and 
Smoking and forced expiratory flows in men handling barley grain

\begin{tabular}{lcccccc}
\hline & $\begin{array}{c}P F \\
<70 \% \text { pred }\end{array}$ & $\geqslant 70 \%$ pred & $\begin{array}{c}\dot{V}_{\text {s0 }} \\
<70 \% \text { pred }\end{array}$ & $\geqslant 70 \%$ pred & $\begin{array}{c}\dot{V}_{75} \\
<0 \% \text { pred }\end{array}$ & $\geqslant 70 \%$ pred \\
\hline Smokers & 10 & 34 & 23 & 21 & 36 & 8 \\
Ex-smokers & 1 & 19 & 8 & 12 & 17 & 3 \\
Non-smokers & 1 & 15 & 2 & 14 & 6 & 10 \\
Total & 12 & 68 & 33 & 47 & 59 & 21 \\
\hline
\end{tabular}

four loading a non-dusty cargo. Measurements were made before starting work and then every two hours until the end of the shift. Four of the men handling barley wore $3 \mathrm{M}$ non-toxic particle masks and two wore Racal air stream helmets. Four were smokers and two had never smoked.

\section{RESULTS}

The six men working with barley had falls in their ventilatory capacity of up to $800 \mathrm{ml}$, whereas the men loading a non-dusty cargo had slight increases over the two days. The four smokers had lower than predicted $\dot{\mathrm{V}}_{50 \mathrm{~s}}($ range $41 \%-60 \%)$ and $\dot{\mathrm{V}}_{75 \mathrm{~s} .}(25 \%-$ $30 \%)$; in one of the two non-smokers the $\nabla_{75}$ only was low (33\% predicted).

Figure 1 shows the flow volume curves at the beginning and end of the second day's shift for the men who were exposed to barley. The curves are lined up at residual volume and the $50 \%$ and $75 \%$ marks are of the initial FVC. Worker B who had not previously reported work related respiratory symp- toms had a reduction in $\mathrm{FEV}_{1}$ and FVC but there was no change in the shape of the flow volume curve. The curves of the other men became more concave after exposure, with falls in $\dot{\mathrm{V}}_{50}$ but little or no change in $\bar{V}_{75}$. Workers $B$ and $D$ showed the least change in the shape of their flow volume curves, possibly because they used airstream helmets.

\section{Previously unexposed subjects}

Five volunteers without respiratory disease sat in the dustiest part of the dock silo for two hours while barley was being loaded. The respirable dust concentration measured with a Casella type 113A gravimetric sampler was $31 \mathrm{mg} / \mathrm{m}^{3}$. Flow volume spirometry was performed before exposure, immediately after exposure, and at intervals throughout the next three days. Specific conductance and lung volumes were measured by plethysmography on the afternoon before exposure and on the afternoons of the exposure day and the next day.
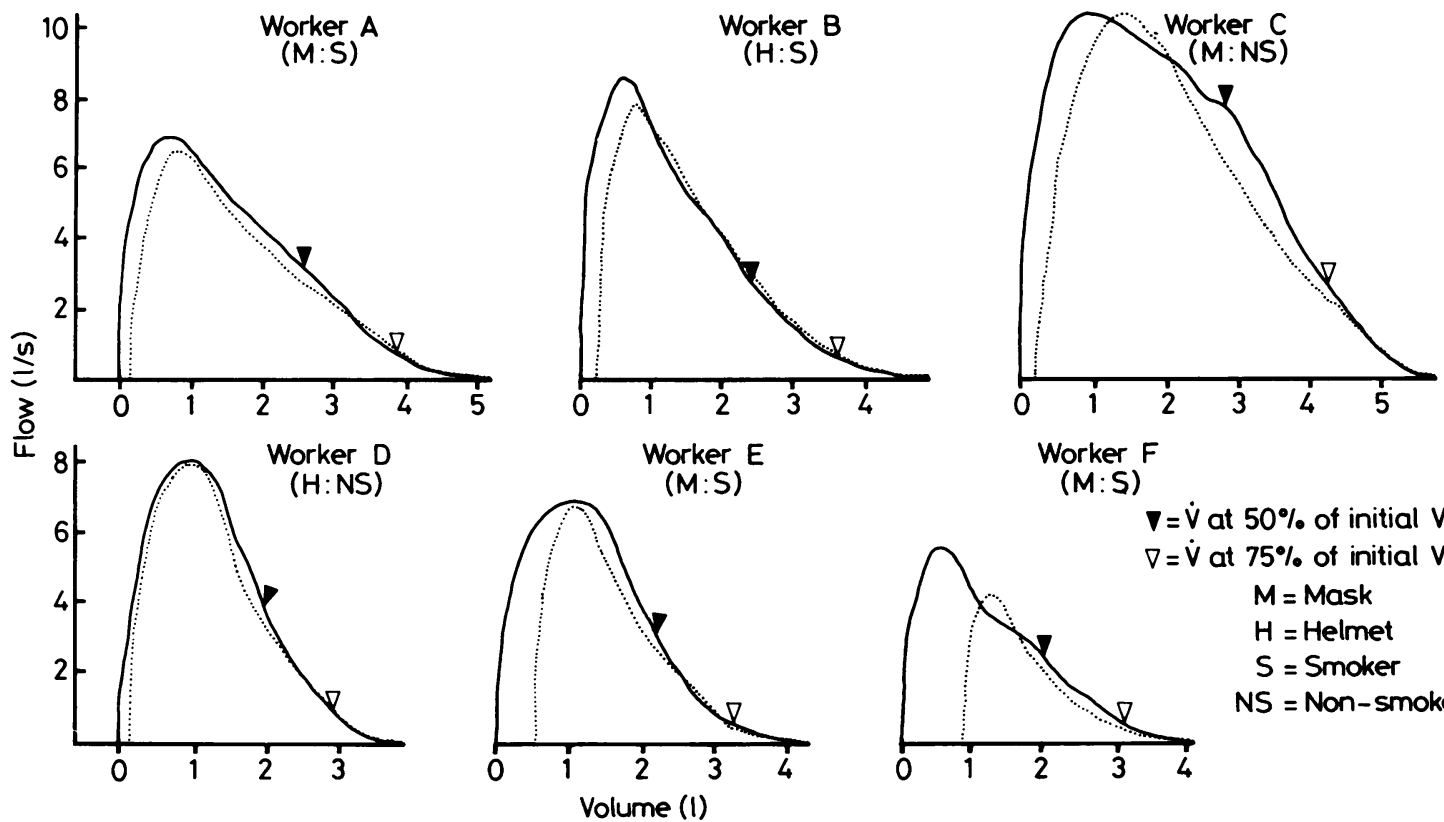

$(\mathrm{M}: \mathrm{S})$

$\nabla=\dot{V}$ at $50 \%$ of initial VC $\nabla=\dot{V}$ at $75 \%$ of initial VC

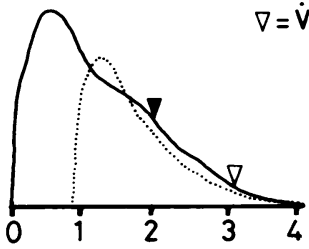

$M=$ Mask

$\mathrm{H}=$ Helmet

$\mathrm{S}=$ Smoker

NS $=$ Non-smoker

Fig 1 Flow volume curves at the beginning (-) and end (...) of the across shift measurements. 
(a) Subject 1

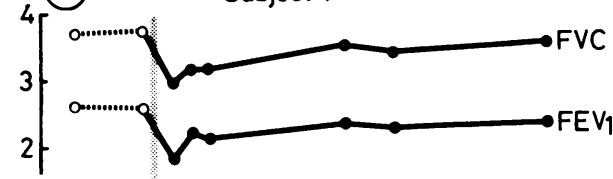

아

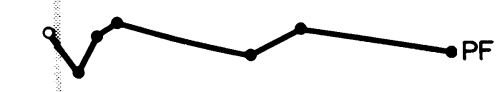

$\longrightarrow \dot{V}_{50}$
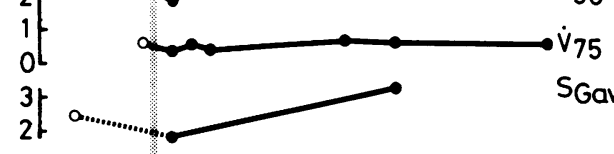

5f $\mathrm{O}$..................... $\mathrm{TLC}$
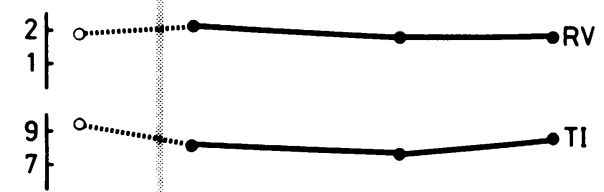

2.0 1.5

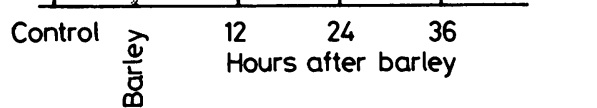

(b)

Subject 3
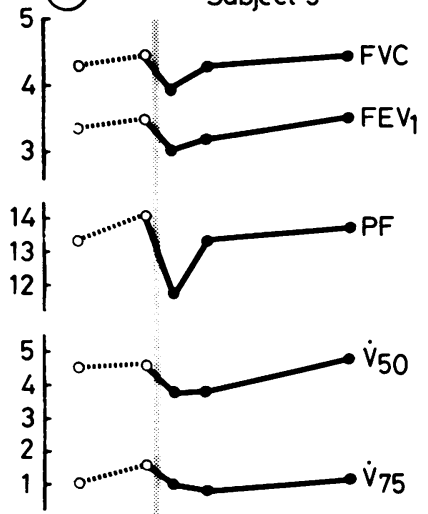

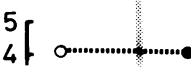

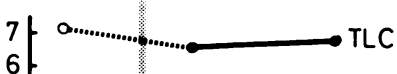

3.

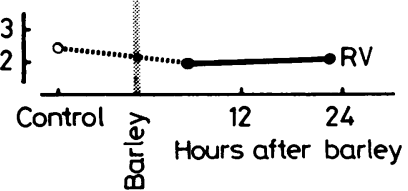

$2^{3}$ Subject 2
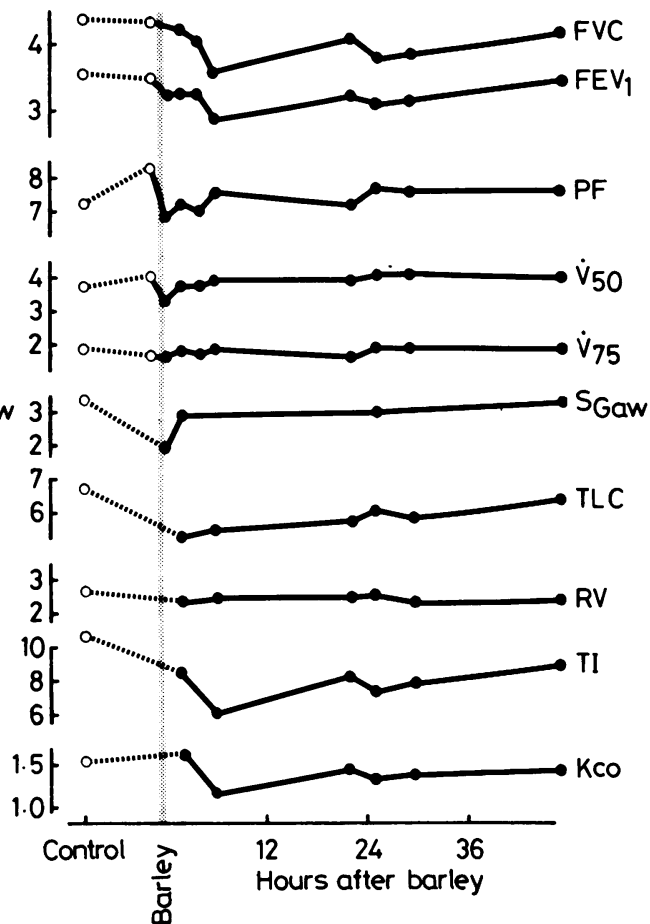

Subject 4

Subject 5
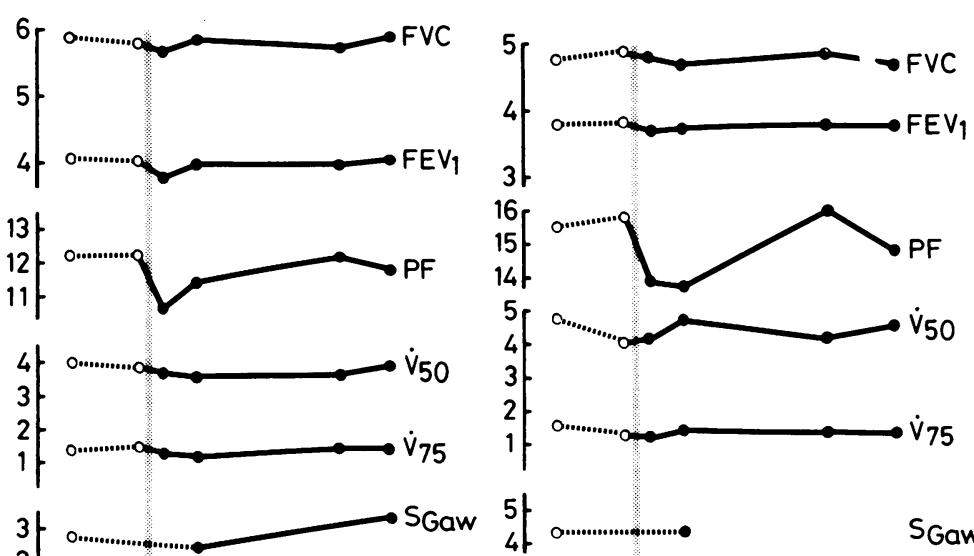

SGaw
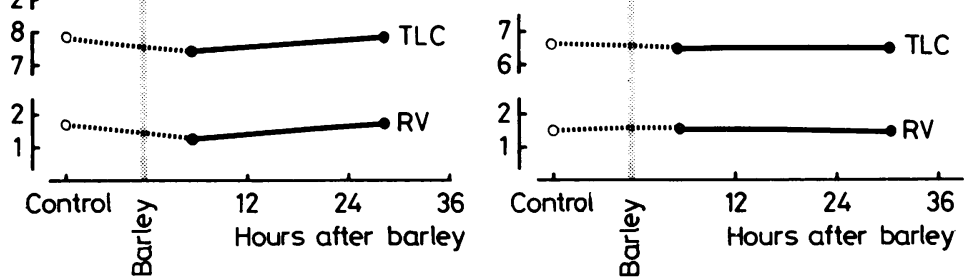

Fig 2a and b Lung function changes in five normal subjects after exposure to barley dust. Units: $F E V_{1}(1), F V C(1)$, $T L C(1), R V(1) ; P F(l / s), \dot{V}_{s 0}(l / s), \dot{V}_{75}(l / s) ; S_{G a w}\left(s^{-1} \mathrm{kPa}^{-1}\right) ; \mathrm{Tl}\left(\mathrm{mmol}^{-1} \mathrm{~min}^{-1} \mathrm{kPa}\right) ; \mathrm{Kco}\left(\mathrm{mmol}^{-1} \mathrm{~min}^{-1} \mathrm{kPa}^{-1} l\right)$. 


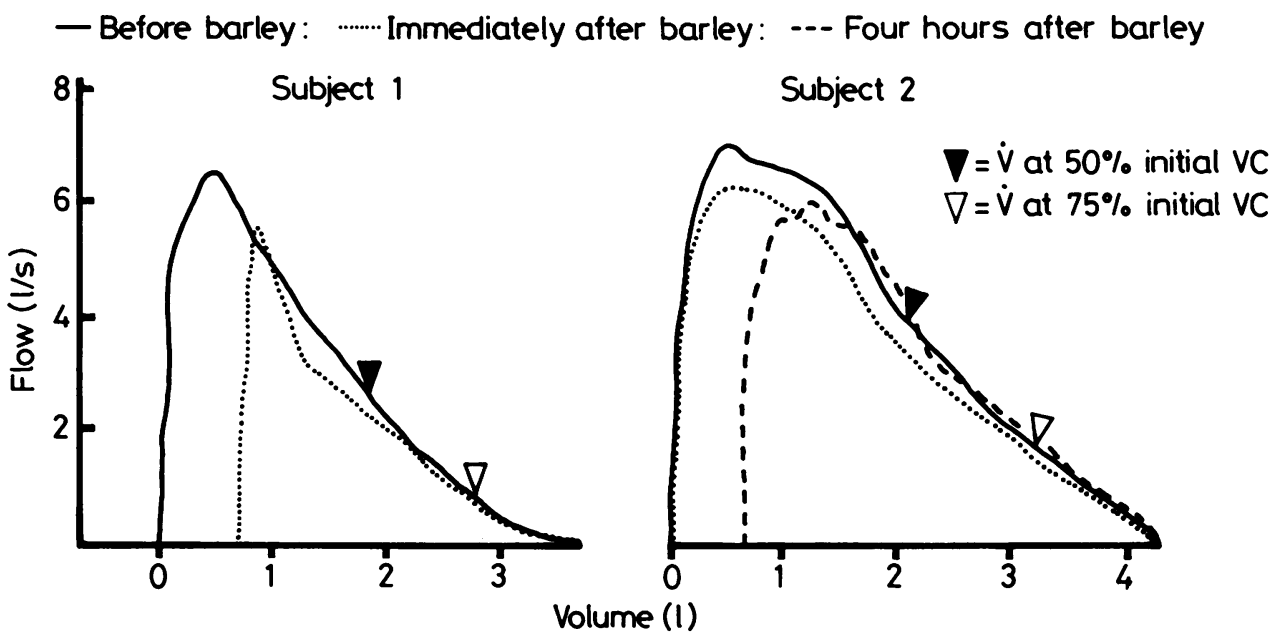

Fig 3 Flow volume curves in subjects 1 and 2 before and after exposure to barley dust.

The two subjects with the largest response repeated the exposure to investigate possible changes in transfer factor for carbon monoxide ( $\mathrm{Tl})$, measured by the single breath technique, and lung volumes, measured by helium dilution.

\section{RESULTS}

The lung function results of all five volunteers are shown in figs $2 \mathrm{a}$ and $2 \mathrm{~b}$. Subjects 1 and 2 showed the largest decreases in $\mathrm{FEV}_{1}$ and $\mathrm{FVC}$ which remained low, as did the TLC and Tl, after the other lung function indices had returned to normal levels for these subjects. The peak flow, $\dot{\mathrm{V}}_{50}$ and $\dot{\mathrm{V}}_{75}$ were low-

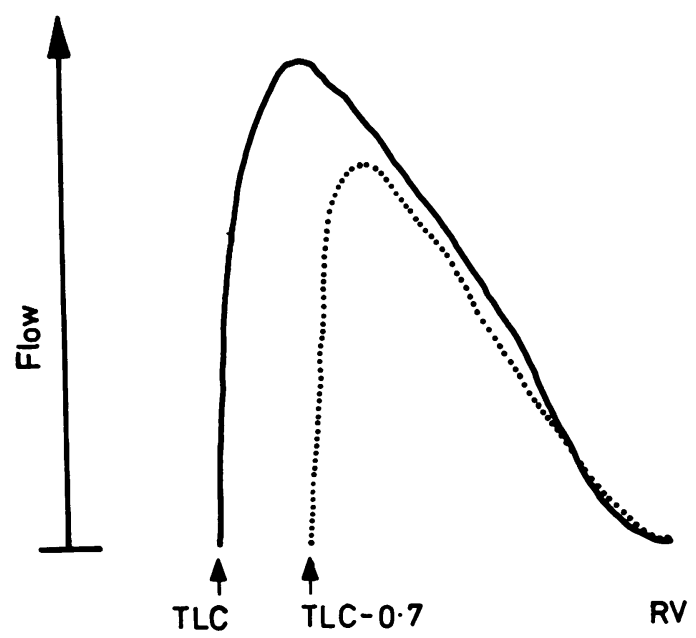

Fig 4 Flow volume curves from different inspiratory volumes. est immediately after exposure and had regained their normal levels by the next afternoon, as had the specific conductance. The changes in total lung capacity were of similar magnitude to those in the FVC. In subjects 1 and 2 the FEV, and FVC which remained below normal for up to 72 hours showed a cyclical pattern of improvement overnight and decline during the next day and evening. Subject 2 inhaled a dose of salbutamol on the second evening, which produced a transient increase in $\mathrm{FEV}_{1}$ and FVC of about $300 \mathrm{ml}$ (not shown in figure). In subject 2 the $V_{50}$ and $V_{75}$ were reduced immediately after exposure before there was any significant change in the FVC (fig 3). Later, when the lowest FVC was recorded, the flow rates in the latter part of the flow volume curve were at pre-exposure levels.

In all subjects the maximum lung function changes coincided with systemic symptoms and fever as previously reported. ${ }^{4}$

\section{Discussion}

Although the work force had "normal" ventilatory capacity, many of the men had small airways function well below $70 \%$ of that predicted, and this occurred more frequently in the smokers. Such a deficiency in grain workers who smoked has also been reported by Cotton et al. ${ }^{7}$

Two flow volume curves from different inspiratory levels on a subject with no dust exposure are shown in fig 4 and they show that there is no change in the end of the curve when the expiration is begun from less than TLC. The curves obtained before and after exposure to barley dust in the dockworkers (fig 1) and in the previously unexposed subjects 
(fig 3) are similar to these, suggesting that the major change is in inspiratory capacity. Interestingly, all the normal subjects complained, sometimes bitterly, of discomfort on inspiration after exposure to barley. The fall in transfer factor was mainly due to a reduction in alveolar volume, since there was little or no change in Kco. The peak flow was lowest immediately after exposure and had partly recovered within two hours. At this time the $S_{\mathrm{Gaw}}$ was measured and was also reduced. Had it been practicable to measure $\mathrm{S}_{\mathrm{Gaw}}$ immediately after exposure a greater decrease might have been found. These short lived changes were possibly due to the irritant effect of the high dust concentration on the large and medium sized airways.

McDermott et al reported similar results when normal subjects were exposed to cotton dust ${ }^{8}$; the airways resistance returned to the initial value

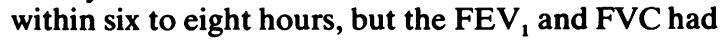
not fully recovered $48-72$ hours later. No change was found in transfer factor after exposure to cotton dust. The systemic symptoms were similar to those found in the present study, as was the pattern of deterioration in ventilatory capacity during the following evenings, with recovery overnight.

The reduction in inspiratory capacity is difficult to explain. It is unlikely that it is due to weakness of the chest wall muscles as there appeared to be no change in the expiratory effort as is shown by the flow volume curves, in particular the peak flow, and there were no complaints of weakness in other parts of the body.

The small response to salbutamol 48 hours after exposure is consistent with a continuing production of a bronchoconstrictor substance for the small airways; at this time there was no narrowing of the large and medium sized airways.

The lung function changes are similar to those seen in acute interstitial inflammation with involvement of the small airways. The reaction appears to be insufficient to produce a measurable reduction in transfer factor. To investigate this further it would be necessary to measure compliance and recoil pressure, and it is planned to do so in future studies.
A possible explanation for the effect of barley (and of cotton) is that these dusts may cause the release of mediators such as leukotrienes in the lung. Weiss and co-workers ${ }^{9}$ and Griffin and co-workers ${ }^{10}$ have shown the bronchoconstrictor effect of inhaled leukotrienes in normal and asthmatic subjects. These effects lasted longer than those of inhaled histamine, and possibly the slow absorption of an agent from the retained barley dust in the lungs could cause the release of leukotrienes over an even longer period.

We thank all the men who took part in the study and the British Waterways Board and other dock employers for their cooperation. We are grateful to Dr RC Brown for his suggestions regarding leukotrienes.

\section{References}

' Broder I, Mintz S, Hutcheon M, et al. Comparison of respiratory variables in grain elevator workers and civic outside workers of Thunder Bay, Canada. Am Rev Respir Dis 1979;119:193203.

${ }^{2}$ Dosman JA, Cotton DJ, Graham BL, Li K-YR, Froh F, Barnet GD. Chronic bronchitis and decreased forced expiratory flow rates in lifetime non-smoking grainworkers. Am Rev Respir Dis 1980;121:11-6.

${ }^{3}$ Kleinfeld M, Messite J, Swencicki RE, Shapiro J. A clinical and physiologic study of grain handlers. Arch Environ Healtho 1968; 16:380-4.

${ }^{4}$ Cockcroft AE, McDermott M, Edwards JH, McCarthy PE Grain exposure - symptoms and lung function. Eur $J$ Respir Dis 1983;64:189-96.

${ }^{5}$ McDermott M, McDermott TJ. Digital incremental techniques applied to spirometry. Proceedings of the Royal Society of Medicine 1977; 70:169-71.

- Cotes JE. Lung function. Assessment and application in medicine. 4th ed. London: Blackwell Scientific Publications, 1979.

7 Cotton DJ, Graham BL, Li K-YR, Froh F, Barnet GD, Dosman JA. Effects of smoking and occupational exposure on peripheral airway function in young cereal grain workers. $\mathrm{Am}$ Rev Respir Dis 1982;126:660-5.

${ }^{8}$ McDermott M, Skidmore JW, Edwards JH. The acute physiological, immunological and pharmacological effects of inhaled cotton dust in normal subjects. Medicine de Empressa 1969; 5: 138-9.

' Weiss JW, Drazen JM, Coles N, et al. Bronchoconstrictor effects of leukotriene $C$ in humans. Science 1982;216:196-8.

${ }^{10}$ Griffin M, Weiss JW, Leitch AG, et al. Effects of leukotriene D on the airways in asthma. $N$ Engl $J$ Med 1983;308:436-9. 